\title{
Enhancement Opportunities for Conceptual Design in Aerospace Based on the Advanced Morphological Approach
}

\author{
Vladislav T. Todorov ${ }^{1, *}$, Dmitry Rakov ${ }^{2} \mathbb{D}$ and Andreas Bardenhagen ${ }^{1} \mathbb{D}$
}

1 Aircraft Design and Aerostructures, Institute of Aeronautics and Astronautics, Technische Universität Berlin, 10587 Berlin, Germany; andreas.bardenhagen@tu-berlin.de

2 Blagonravov Mechanical Engineering Research Institute (IMASH), Russian Academy of Sciences, 101990 Moscow, Russia; rdl@mail.ru

* Correspondence: vladislav.t.todorov@tu-berlin.de

check for

updates

Citation: Todorov, V.T.; Rakov, D.;

Bardenhagen, A. Enhancement

Opportunities for Conceptual Design in Aerospace Based on the Advanced Morphological Approach. Aerospace 2022, 9, 78. https://doi.org/10.3390/ aerospace 9020078

Academic Editors: Spiros Pantelakis, Andreas Strohmayer and Liberata Guadagno

Received: 15 December 2021

Accepted: 27 January 2022

Published: 1 February 2022

Publisher's Note: MDPI stays neutral with regard to jurisdictional claims in published maps and institutional affiliations.

Copyright: (c) 2022 by the authors. Licensee MDPI, Basel, Switzerland. This article is an open access article distributed under the terms and conditions of the Creative Commons Attribution (CC BY) license (https:// creativecommons.org/licenses/by/ $4.0 /)$.

\begin{abstract}
The current challenges facing the aerospace domain require unconventional solutions, which could be sought in new configurations of future aircraft and spacecraft. The choice of optimal concepts requires the consideration of a significant amount of competing engineering solutions and takes place under conditions of uncertainty. Such a problem can be addressed by enhancing existing methods for analysis and synthesis solutions, such as the Advanced Morphological Approach (AMA). It uses morphological analysis to provide a more exhaustive overview of possible problem solutions, relies on expert evaluations of alternative technological options and applies clustering to the solution space. Although an intuitive method for structured concept generation, the AMA exposes the need for more robust problem structuring, improved objectivity of options evaluation and accounting for uncertainties. The current article suggests ways to overcome these challenges and their possible integration in the process. In particular, the integration of fuzzy sets is proposed to model uncertainties during the evaluation of technological options by the experts. The Fuzzy Analytical Hierarchy Process is adapted for integration into the AMA and for the conceptual design of aerospace vehicles.
\end{abstract}

Keywords: conceptual design in aerospace; advanced morphological approach; fuzzy sets

\section{Introduction}

The most important stage in the design of innovative aircraft systems is the concept design phase [1]. Mistakes at this stage might lead to significant increases in cost and lead time and could have a fatal impact on the project. The configuration of the aircraft obtained in the conceptual design stage is numerically simulated and modified in the preliminary design. In most cases, preliminary design approaches are based on development trends derived from databases and statistics [2]. However, this is not the case when aiming to develop innovative aircraft configurations, since breakthrough technologies lack experimental and statistical data. The non-metric character of design parameters, as well as the conflicting criteria, represent further challenges of conceptual design. The target function of a potential optimization problem could hardly be solved by common theoretical methods since it is discontinuous; it cannot always be defined; it exists in the operator notation; it is not based on analytical expressions; it is not differentiable, not unimodal, not separable and not additive [3]. Additionally, the circumstances in the aviation domain put forward the necessity for a technological breakthrough. The sector is forced to address the demands for higher efficiency and drastic emission reduction, while no short-term technological answer is yet in sight $[4,5]$.

Along with heuristic methods, morphological analysis (MA) is used during the conceptual design phase [2,6]. It allows the generation of a significantly wider space of possible problem solutions than conventional methods such as brainstorming and helps to avoid the 
aforementioned conceptual design challenges. This is achieved by decomposing the problem into (sub-)functional and characteristic components (denoted as "attributes"). Each attribute is assigned to a set of appropriate technological options (denoted in the following as "options"). The attributes and their corresponding options are organized in a morphological matrix (MM). An example MM excerpt for the conceptual design of an unmanned aerial system is shown in Figure 1. The entity of all possible attribute-option combinations defines the solution space. The significant amount of synthesized configurations in such a way increases the possibility to find the optimal solution even for a non-conventional or demanding problem statement. In such situations, the optimal solutions can be overseen by conventional idea generation methods.

\begin{tabular}{|l|l|l|l|l|}
\hline Lift generation & Option 1 & Option 2 & Option 3 & Option 4 \\
\hline Internal Energy supply & Aerodynamic & Thrust & Aerostatic \\
\hline Power generation & Electric & $\begin{array}{l}\text { Chemical, reversible } \\
\text { (e.g. battery) }\end{array}$ & $\begin{array}{l}\text { Chemical irreversible } \\
\text { (e.g. fuel tank) }\end{array}$ & $\begin{array}{l}\text { Mechanic (e.g. } \\
\text { fly-wheel) }\end{array}$ \\
\hline Fuselage & None & Single fuselage & Twin boom & Rocket engine \\
\hline Altitude control & $\begin{array}{l}\text { Aerodynamic/ } \\
\text { elevators }\end{array}$ & Thrust adjustments & Aerostatic & \\
\hline Wing morphing & No & Yes & & \\
\hline
\end{tabular}

Figure 1. An excerpt from the morphological matrix for the structural synthesis of an unmanned aerial system. Data from ref. [7].

MA has been applied to the conceptual design of aerospace vehicles within the Advanced Morphological Approach (AMA), developed by Rakov and Bardenhagen [7,8]. The method extends the classical MA by integrating qualitative expert evaluations of the options and by offering an intuitive solution space exploration. The AMA steps can be summarized as follows [7-9]: (1) problem statement definition; (2) definition of the MM and a set of evaluation criteria; (3) evaluation of the technological options by domain experts according to each criterion; (4) inclusion of reference solutions based on existing aerospace vehicle configurations; (5) definition of impossible combinations of options for different attributes; (6) generation of the solution space; (7) clustering of the generated solutions based on the given expert evaluations; (8) visualization and reporting.

A simplified diagram of the methodology is presented in Figure 2. From the generated vast solution space in the MM, the method picks a limited amount of superior solutions based on their criteria evaluations $[7,10]$. Hence, the AMA represents a structured way to intuitively find optimal solutions to a given problem and avoid the aforementioned challenges of conceptual design. The application of AMA in the aerospace domain has been demonstrated on the conceptual design of unmanned aerial vehicles [7] and orbital reentry vehicles [11] (Figure 3).

Along with the mentioned benefits, the current AMA comes with a set of challenges and corresponding improvement possibilities, which have been presented by Todorov et al. in [12]. These are namely (a) uncertainty handling, (b) improved problem structuring and (c) multidisciplinary expert judgment elicitation.

The issue of dominating uncertainties (a) within AMA is faced during step 3 of the process: the evaluation of options by domain experts (further referred to as "experts", "decision-makers" or DMs). The method requires the DMs to evaluate each option according to a certain criterion by assigning an integer value from the qualitative scale between 1 (worst) and 9 (best). However, it is not always clear whether the quality of option A is, for example, "very good" or just "good". Such hesitation could result in a deviation of 
several units on the scale from the answer that is "ultimately correct" and thus reduces the reliability of the final results. This uncertainty could further increase due to the fact that the evaluation objects are innovative technologies lacking statistical and experimental data. Although the DMs are domain experts in the best-case scenario, they could naturally also lack precise knowledge on an untested or non-introduced technology.

\begin{tabular}{|l|l|l|l|l|}
\multicolumn{5}{|c}{ Decomposition (MM) } \\
\hline & Opt 1 & Opt 2 & $\ldots$ & Opt n \\
\hline Attr 1 & P 1.1 & P 1.2 & $\ldots$ & P 1.n \\
\hline Attr 2 & P 2.1 & P 2.2 & $\ldots$ & P 2.n \\
\hline$\ldots$ & $\ldots$ & $\ldots$ & $\ldots$ & $\ldots$ \\
\hline Attr k & P k.1 & P k.2 & $\ldots$ & P k.n \\
\hline
\end{tabular}

Morphological matrix \& definitions

- Criteria

- Morphological matrix
Technology evaluation

\begin{tabular}{|l|l|} 
& P i.j \\
\hline Criterion 1 & Eval 1 \\
\hline Criterion 2 & Eval 2 \\
\hline$\ldots$ & $\ldots$ \\
\hline Criterion k & Eval 3 \\
\hline
\end{tabular}

Assessments $0 \ldots 9$

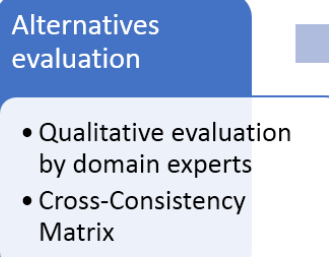

Synthesis

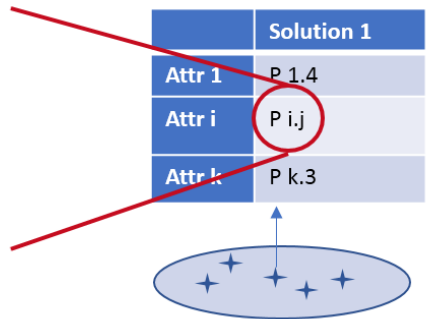

Solution space generation

- Filtering of the impossible solutions

- Addition of existing reference solutions

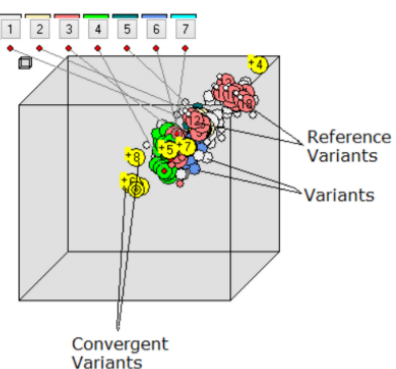

Variants

Clustering and analysis

- Clustering of similar solutions

- Analysis of the relevant clusters and solutions

Figure 2. An overview of the AMA method. Reprinted from ref. [12].
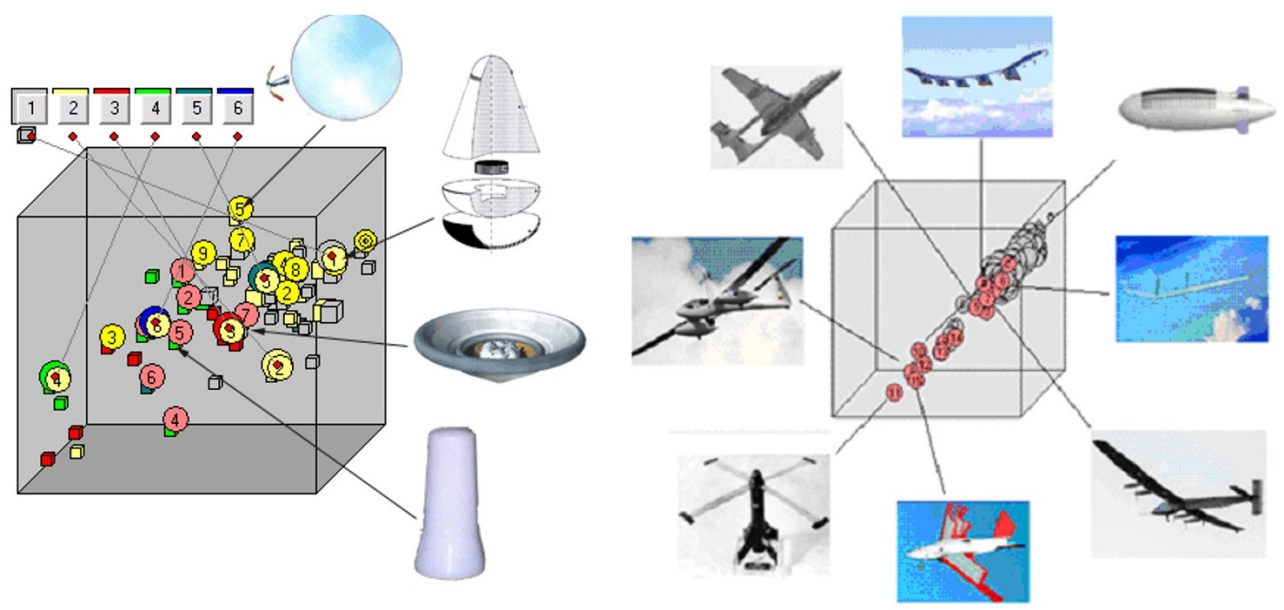

Figure 3. Reference solutions in the morphological solution space for the conceptual design tasks for an orbital reentry vehicle (left) and an unmanned aerial vehicle (right). Reprinted from refs. [7,11].

Further inaccuracy in the AMA outcome could result from the lack of consideration of technological options' interactions. Along with their separate evaluations mentioned above, certain technological combinations may additionally increase the overall value of particular configurations, which should also be reflected in the results. This aspect is acknowledged and is outlined in the future work recommendations for the improvement of the AMA.

Problem structuring (b) becomes an issue when the product to be designed represents a complex engineering solution such as an aerospace vehicle. This case requires a more thorough structural decomposition and the appropriate organization and weighting of components and criteria. This is necessary to properly reflect the complex functional division of sub-systems. The selection of appropriate problem structuring also requires the definition of evaluation aspects such as which evaluation scale to use or whether the evaluations should be absolute or pairwise comparative. Furthermore, the issue is also linked to the choice of an uncertainty modeling method. 
The current article addresses the issues and the improvement possibilities for addressing uncertainties (a) and defining a robust problem structuring (b) for the AMA. Section 2 will first handle the classification of uncertainties and outline which uncertainties one faces during the option evaluations within the AMA. The choice of ordinary or Type 1 fuzzy sets as an approach for addressing the uncertainties among other possibilities is explained. After introducing their general definition, different types of fuzzy sets are briefly summarized. A way to integrate fuzzy numbers in the AMA is then suggested. Section 3 will address the issue of problem structuring. First, a classification of Multiple-Criteria Decision-Making methods is presented and the advantages of using Fuzzy Multiple-Attribute DecisionMaking methods for the enhancement of the AMA are justified. Ultimately, an approach is suggested to incorporate the Fuzzy Analytic Hierarchy Process for the conceptual design of aerospace vehicles with the AMA.

\section{Addressing Uncertainties}

The conceptual design of a new aircraft generation is characterized by a significant amount of unknown information on innovative technologies. At the same time, the decisions made during this design phase also influence the development of the next design stages [7]. For aerospace vehicles, incorrect conceptual decisions can result in project delays and financial drawbacks. For this reason, situations associated with a significant lack of data require the anticipation of possible deviations and thus suitable models to describe uncertainties. This is the case with evaluations of options by expert groups in the AMA.

\subsection{Uncertainty Classification}

The most used taxonomy to classify uncertainties comes from the field of risk assessment and considers two main types: aleatory and epistemic uncertainty [13,14]. Aleatory (also stochastic) uncertainty is defined as the inherent variation of a system or the environment. Although well recognizable, it cannot be reduced or avoided. At the same time, epistemic uncertainty (also cognitive or subjective) results from the inaccurate modeling of a system or a process due to a lack of knowledge. Increasing the available data or knowledge on the matter could thus help to reduce epistemic uncertainty.

Worth noting is also the taxonomy suggested by Thunnissen [14,15], which introduces further types of uncertainty besides the ones already presented. These are the ambiguity in terms of imprecision or vagueness, as well as the uncertainty caused by the interaction of multiple disciplines or events (interaction uncertainty).

When focusing on the design of complex systems such as aerospace vehicles, further uncertainty classes have been suggested that relate rather to more precise modeling of system parameters [14]. These are the uncertainties coming from inaccurate design problem formulations, such as due to the operational environment, measurement, as well as modeling and numerical errors.

However, the conceptual design phase implies the definition of the initial vehicle structure. This task depends vastly on efficient interdisciplinary communication. The "correct" decision-making at this early stage requires proper qualitative modeling of technologies with scarce available knowledge rather than the reduction of measurement or numerical errors. For this reason, one can outline epistemic uncertainty, ambiguity and disciplinary interaction as the primary sources of deviations from the optimal conceptual design of new aerospace vehicle generations. Since the latter two categories involve a lack of knowledge and sub-optimal information exchange, these will be also considered as epistemic uncertainties in the current article.

This classification helps to identify the types of uncertainties that need to be addressed or at least acknowledged within the AMA method. Potential sources of uncertainties can be found in the evaluation of technological options. Particularly, subjective results could be obtained in the following cases:

1. When an expert estimates the quality of an option according to a certain criterion. Subjective assessments can be due to the following drawbacks: 
- $\quad$ Epistemic uncertainty—the lack of knowledge on untested or non-introduced technologies;

- Cognitive bias-the unconscious heuristics people use to solve uncertain tasks, which may lead to systematic errors [16].

2. During group discussions of a certain technology by experts from different expertise domains (disciplinary interaction).

3. As a result of aggregation of multiple expert opinions-be it behavioral (group discussions) or mathematical.

The current article suggests that epistemic uncertainty during option evaluation could be accounted for by introducing an appropriate modeling approach, which will be discussed in the following. Handling cognitive bias, disciplinary interaction and assessment aggregation from multiple experts could be considered as research topics linked to the development, structuring and conduction of the expert workshops and are not discussed in the present work.

\subsection{Uncertainty Modeling Approaches}

The main requirement for the modeling of epistemic uncertainty is the ability to quantify and map the uncertain areas during decision-making onto an intuitive and robust process. It is thus necessary to find a proper mathematical and computational representation of this uncertainty type.

Zang et al. [17] summarize three ways to represent uncertainties in a computer code used for modeling and simulation. These are the interval bound, the membership function and the probability density function (PDF), shown in Figure 4. The methods incorporate different theories to describe uncertainties. Based on the classical probability theory, the PDF offers uncertainty modeling with the highest detail among the three mentioned options. However, it hides certain drawbacks when dealing with problem statements lacking statistical data [18]. Firstly, the choice of a suitable probability distribution requires prior experimental data. Secondly, PDFs aim to "represent one's degree of belief that a specific realization of a parametric value, physical process, or event can occur" [18] (p. 2). This type of uncertainty modeling has already been applied in the aerospace domain. The works of Monroe [19] and Unal et al. [20] describe an expert judgment elicitation methodology for the conceptual design of innovative launch vehicles. They use qualitative expert input in order to quantify the uncertainties through probability distributions. The parameters that were estimated by the experts referred to deterministic characteristics such as system weight and cost. Since the technologies used for the design in those works were supposed to be "extrapolated" from existing systems of that time [20], one could assume that the aerospace experts involved had some initial experience and statistical data on the technologies to lean on.
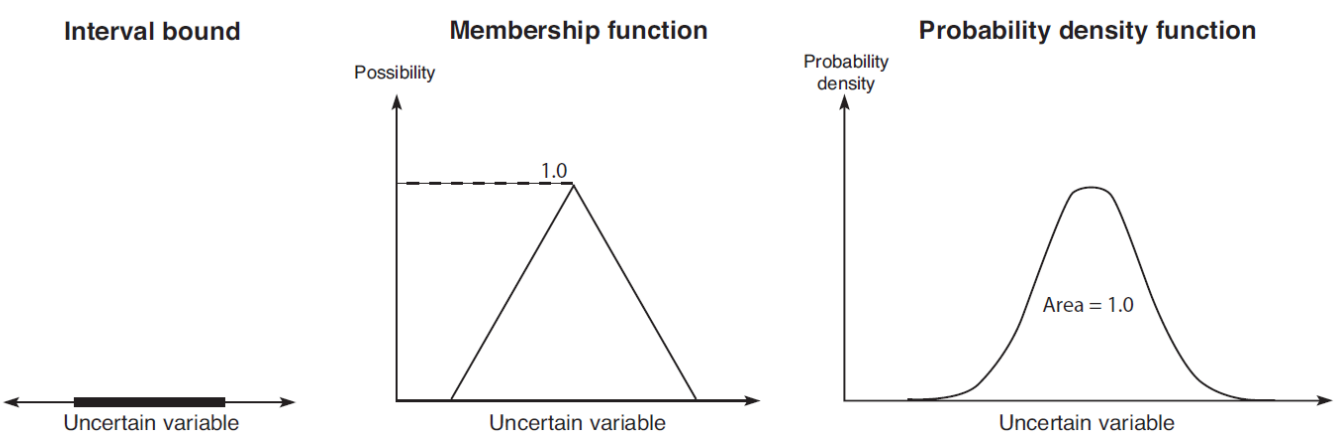

Figure 4. Ways to describe uncertainty. Reprinted from ref. [17].

However, the AMA strives not only to include technological options derived from ones already in operation, but also to consider technologies lacking any prior experimental data. Therefore, the uncertainties to be modeled are not referred to the concrete estimation 
of aircraft characteristics such as weight or certain flight qualities but rather a higher-level qualitative comparison of more abstract combinations of technological options.

At the same time, the interval bound defines solely the upper and lower limits of the possible value range and offers a very simplified approximation [17].

The membership function is a mathematical tool used in the fuzzy logic and fuzzy set theory to describe fuzzy sets, introduced by Lotfi Zadeh in 1965 [21], and is "particularly well-suited for handling incomplete information, the unsharpness of classes of objects or situations, or the gradualness of preference profiles" [22] (p. 4). This approach allows us to mathematically model events or statements, which can be semantically assigned to multiple parameter values simultaneously $[21,22]$. Such a philosophy could be used to represent subjective, ambiguous and vague statements made by experts for decision-making tasks during early conceptual design, which corresponds to the evaluation of options within the AMA.

\subsection{Fuzzy Sets}

An ordinary (so-called Type 1) fuzzy set $\tilde{A}$ is formally defined by a given set $X$ and a membership function $\mu(x)$, which maps every $x \in X$ to a real value from the interval $[0,1]$ (Equations (1) and (2)) [22,23]. The function reflects the "grade of membership" of each $x$ in $\tilde{A}$.

$$
\begin{gathered}
\tilde{A}=\left\{\left(x, \mu_{\tilde{A}}(x)\right) \mid x \in X\right\} \\
\mu_{\tilde{A}}(x): X \rightarrow[0,1]
\end{gathered}
$$

Such a definition allows to model uncertainties by assigning a suitable membership function to an unsharp linguistic statement. This is achieved by considering the "level of truth" or the "grade of membership" of the statement for each parameter value $x$. For example, one could use fuzzy sets to represent the vague definition "wise" by subjectively assigning it to a person's age, as depicted in Figure 5. Since there exists no definite age threshold between defining a person as "wise" and "not wise", the fictional DM has decided to place ages between 30 and 75 in an uncertain area, where wisdom is accumulated nonlinearly. People aged 75 and older are assumed by this DM to fully correspond to the definition of "wise" with full membership of $\mu(75 \leq x)=1$.

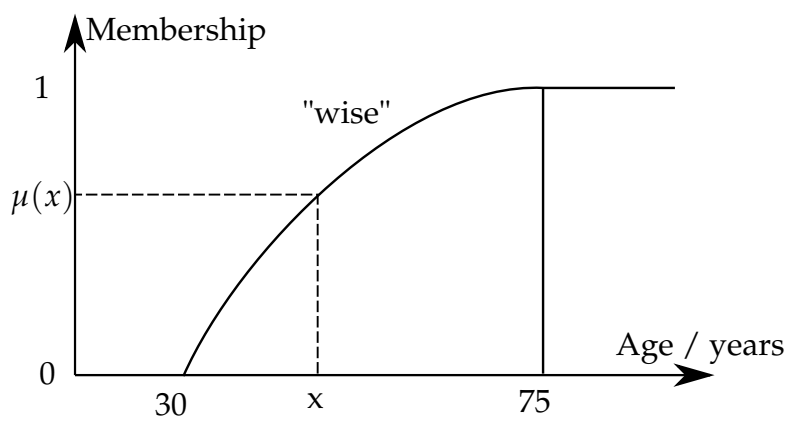

Figure 5. A fuzzy representation of the vague adjective "wise" referring to a person's age.

The last several decades have seen the active development of fuzzy sets through extensions and generalizations of the presented ordinary case. As a result, multiple new types of fuzzy sets have been introduced and have found applications in numerous domains [23]. A brief overview of the most used types is shown in Table 1. 
Table 1. A summary of some of the most used fuzzy set types.

\begin{tabular}{lll}
\hline Name & Definition & Description \\
\hline Type 2 fuzzy sets [21,23] & $\tilde{A}=\left\{\left((x, u), \mu_{\tilde{A}}(x, u)\right) \mid 0 \leq \mu_{\tilde{A}}(x, u) \leq 1\right\}$ & The membership function is fuzzy itself \\
\hline & $\tilde{A}=\left\{\left\langle x, \mu_{\tilde{A}}(x), v_{\tilde{A}}(x)\right\rangle ; x \in X\right\}$ & \\
& $\begin{array}{l}\mu_{\tilde{A}}: X \rightarrow[0,1] ; v_{\tilde{A}}: X \rightarrow[0,1] \\
\text { Intuitionistic fuzzy sets [24] } \leq \mu_{\tilde{A}}+v_{\tilde{A}} \leq 1\end{array}$ & $\begin{array}{l}\text { Introduction of the non-membership } v_{\tilde{A}}(x) \text { and } \\
\text { the hesitation margin } \pi_{\tilde{A}}[24,25]\end{array}$ \\
& $\pi_{\tilde{A}}(x)=1-\mu_{\tilde{A}}(x)+v_{\tilde{A}}(x)$ & The function $h_{\tilde{A}}(x)$ returns a subset of $[0,1]$ \\
\hline \multirow{2}{*}{ Hesitant fuzzy sets [26] } & $\tilde{A}=\left\{\left\langle x, h_{\tilde{A}}(x)\right\rangle \mid x \in X\right\}$ & \\
\hline
\end{tabular}

The common aspect of the given fuzzy set types is that they all introduce an additional "grade of freedom" or dimension of fuzziness to the already existing membership. One could interpret it as the uncertainty in the position of the membership value for each $x$. The corresponding authors justify the necessity for the additional uncertainty level with the possible hesitation of the DM when choosing the membership value $\mu_{\tilde{A}}(x)$ for each particular element $x$. Kahraman et al. [27] outline multiple applications of the intuitionistic and hesitant fuzzy sets within Fuzzy Multiple-Criteria Decision-Making methods.

The mentioned classification of fuzzy sets combines sophisticated ways to model multilayer uncertainty, which vary in their definitions and depth of uncertainty representation. Therefore, it is necessary to pick the most suitable type for the particular purpose in the first place. Secondly, one should carefully evaluate whether complex and overly abstract uncertainty modeling would impact:

1. the comprehensibility of the evaluation process/the questionnaires for the experts, which could reduce the results' reliability

2. the transparency of the scientific method, thus making difficult the recreation of the process and the results.

\subsection{Integration of Fuzzy Sets into the Advanced Morphological Approach}

When incorporating uncertainties into the AMA for the design of aerospace vehicles, one should consider the specificity of the experts usually involved in this type of decisionmaking task. In particular, this tends to be a multidisciplinary group of DMs from different domains who do not necessarily have the deep statistical background encompassing the special characteristics of complex fuzzy sets. Hence, one should find a balance between the optimal capturing of uncertainty and not over-complicating the evaluation task, which could lead to even greater unexplained uncertainty in the results.

For this reason, the authors suggest to start initial studies in such a context by using simpler fuzzy sets (e.g., the ordinary one) and, if necessary, gradually increasing their complexity while studying the experts' perception.

The implementation of fuzzy sets is suitable for the mathematical and computational representation of vague expert statements or evaluations during aircraft conceptual design. At this point, an approach is suggested for the initial integration of ordinary Type 1 fuzzy sets within the AMA. It implies that the expert evaluations of a given technology could be described as membership functions. For this purpose, trapezoidal fuzzy numbers are used, which were defined in $[28,29]$ and are graphically depicted in Figure 6. Consider the definition of a trapezoidal fuzzy number by its four distinctive points: $\tilde{A}=(k, l, m, n)$. These allow the DMs to divide their evaluation into two domains:

1. An interval of full certainty $[l, m]$ with membership $\mu_{\tilde{A}}(x)=1$. The DM gives the highest confidence that this qualitative interval reflects the priority of the option according to a certain criterion as the best.

2. Uncertain intervals $[k, l)$ and $(m, n]$ with membership $0<\mu_{\tilde{A}}(x)<1$. The DM expresses hesitation about these quality values of the option. 
Such a representation of the evaluations will be integrated into a suitable MultipleAttribute Decision-Making method in the next section.

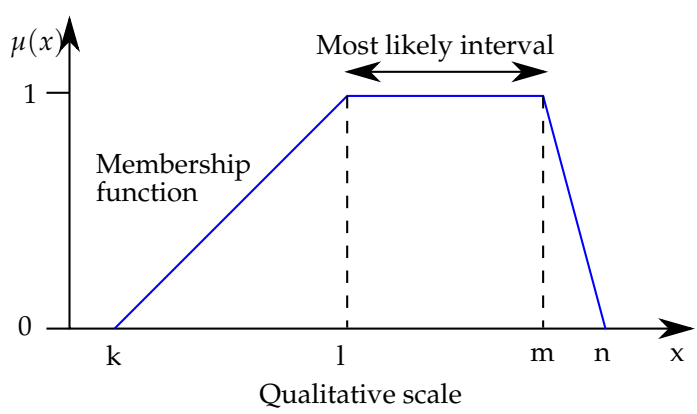

Figure 6. Own depiction of a trapezoidal fuzzy number.

\section{Structuring the Evaluation Process through Hierarchies}

The structural synthesis of promising engineering solutions through the AMA implies problem decomposition and the evaluation of technological options. Aerospace vehicles represent complex systems that can be defined by numerous criteria and subsystem levels. In this case, the appropriate robust structuring and organization of the system decomposition and the option evaluations represent a challenging task. It requires the ranking of discrete technological options of the attributes in order to achieve a higher-level ranking of the entire set of alternative problem solutions (in this case, aircraft configurations). The methods addressing such challenges are classified as Multiple-Attribute DecisionMaking (MADM) algorithms. Following a brief overview of MADM methods, the Analytic Hierarchy Process and its potential contribution to the enhancement of the AMA process will be introduced. Furthermore, possibilities to use fuzzy logic within these methods will be investigated.

\subsection{Overview of Multi-Attribute Decision-Making Algorithms}

Within the domain of operations research (also called decision science), Multi-Criteria Decision-Making (MCDM) is a field that aims to offer mathematical tools for complex decision-making tasks [30]. Two main groups of MCDM methods are distinguished: Multiple-Objective Decision-Making (MODM) and Multiple-Attribute Decision-Making (MADM). While the first strives to solve problems containing continuous variables, MADM addresses tasks where the alternative scenarios represent a discrete decision space [30]. At the same time, these categories can be divided into methods using deterministic data, denoted as crisp methods, and such that take into account uncertainties of human evaluations, or fuzzy ones. Accordingly, the latter are marked as Fuzzy MODM (FMODM) and Fuzzy MADM (FMADM) methods. The nature of the AMA implies the definition of a discrete decision space in the form of a morphological matrix. Furthermore, one of the aims of the AMA enhancement is to account for uncertainties as well. These aspects require the application of a Fuzzy MADM approach. For a more detailed overview of crisp MCDM methods, the reader is encouraged to refer to the corresponding summary under the edition of Figueira et al. [31].

The definition of a typical MCDM method consists of three main elements [30]:

- Alternative actions/options-these correspond to the technological options for each attribute in the AMA.

- A set of criteria - for the AMA, these are the criteria against which the technological options are evaluated.

- A problematic type-one distinguishes among the following problematic types: description, choice, sorting and ranking. The expected outcome of the AMA option evaluation step is to obtain a reliable comparison of the technological options. Hence, this case corresponds to the ranking problematic. 
An extensive overview of existing FMADM methods is given by Kahraman et al. [27] as well as by Chen et al. [28]. In the majority of cases, FMADM methods have been derived from crisp MADM algorithms. Some of the most prominent MADM methods for both crisp and fuzzy evaluations are classified as outranking (ELECTRE, PROMETHEE), distancebased (VIKOR, TOPSIS) and pairwise comparisons-based (Analytic Hierarchy Process, MACBETH) [27].

\subsection{Analytic Hierarchy Process}

The AMA enhancement seeks an intuitive way to structure a complex multi-layer engineering problem statement by considering multiple potentially contradicting criteria. In this context, the Analytical Hierarchy Process (AHP) is used to handle this challenge. The method and its potential application within the AMA will be presented in the following.

The AHP has been developed by Saaty [32] for the purpose of qualitative decisionmaking based on relative pairwise comparisons of alternative options/scenarios [28,32]. One of its main features is the organization of the problem structure in a hierarchical manner. An example of a hierarchy to select an airline to fly with based on the criteria ticket price, airline timetable and offered comfort is shown in Figure 7.

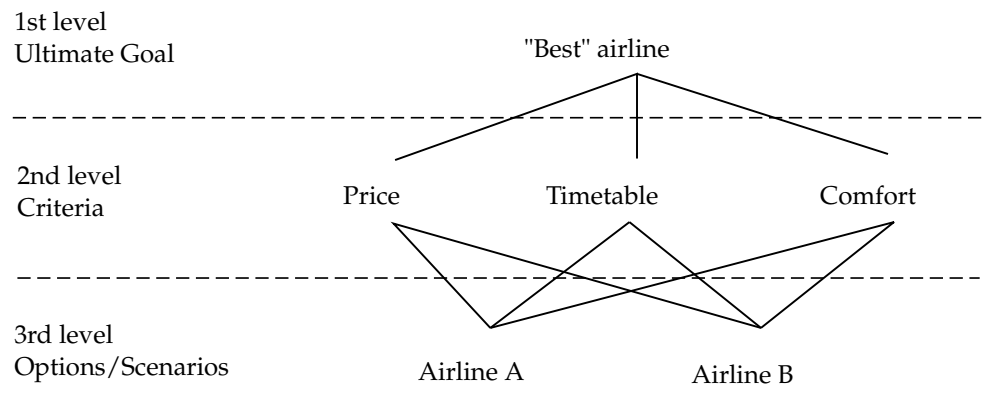

Figure 7. An exemplary hierarchy structure for the selection of an airline to fly with.

Each hierarchy level is considered as a criterion or purpose, according to which the elements of the lower level are evaluated [32]. The evaluations of alternative options from each single level are defined in a relative pairwise manner instead of as absolute values. Hence, each evaluation represents a linguistic statement on the quality of option A referred to option B against a certain criterion, e.g., option A can be much better/slightly better/equal to/slightly worse/much worse than option B. For this purpose, a scale between 1 and 9 with the following legend is used: (1) equality; (3) weak superiority; (5) strong superiority; (7) significant superiority; (9) absolute superiority. The even numbers in between stand for intermediate values. Inferiority is represented by the corresponding reciprocal values. Accordingly, the quality of option B relative to option A is then defined as the reciprocal value of the evaluation from the previous example. Such evaluations for all items of the same level according to a certain criterion are summarized in a reciprocal matrix, represented in Equation (3) [32]. Its elements are the pairwise comparisons $a_{i j}=\frac{\omega_{i}}{\omega_{j}}$, which are obtained from the expert evaluations.

$$
D=\left[\begin{array}{cccc}
\omega_{1} / \omega_{1} & \omega_{1} / \omega_{2} & \ldots & \omega_{1} / \omega_{n} \\
\omega_{2} / \omega_{1} & \omega_{2} / \omega_{2} & \ldots & \omega_{2} / \omega_{n} \\
\ldots & \ldots & \ldots & \ldots \\
\omega_{n} / \omega_{1} & \omega_{n} / \omega_{2} & \ldots & \omega_{n} / \omega_{n}
\end{array}\right]
$$

The next step implies using these relative evaluations $a_{i j}$ in order to find the absolute weights $\omega_{i}$ of the items for the current hierarchy level [32]. In the initial crisp AHP, Saaty showed that these absolute weights correspond, with some approximations, to the dimension values of the main eigenvector of the mentioned reciprocal matrix. It can be obtained by using the so-called "eigenvector method", described by Saaty. 
After establishing the absolute weights of the elements from each single hierarchy level separately, one can proceed with finding the importance of any single element of one level with respect to any element from a higher one. Considering a three-level hierarchy as in Figure 7 , let $\omega_{i j}$ be the weight of the $i$-th element from level 3 with respect to criterion $j$ from level 2 and $b_{j}$ be the weight of the $j$-th element from level 2 with respect to the ultimate goal from level 1 . Then, the importance $U_{i}$ of the $\mathrm{i}$-th element from level 3 with respect to the ultimate goal can be derived as the weighted sum from Equation (4) [28,32]:

$$
U_{i}=\sum_{j=1}^{n} b_{j} \omega_{i j}
$$

These values can be ultimately used to rank the elements from any level according to an element from any higher one.

\section{Integration of a Fuzzy Analytical Hierarchy Process into the Advanced Morphological Approach}

It is worth noting that Saaty himself stated that the crisp AHP already integrated a fuzzy way of thinking in some form-namely by giving the decision-makers (DMs) the opportunity to give relative option evaluations on a qualitative scale [29]. However, DMs could find themselves in situations where they cannot clearly assign a concrete statement to the technology in question [29], e.g., is technology A definitely "much better" or just "slightly better" compared to technology B? This hesitation represents the epistemic uncertainty within the expert evaluations. Instead of being classified as unnecessary and/or incomplete information, the vagueness of the expert opinions should be considered as an integral part of the detailed decision-making process due to its potential impact on the final result. Although the certainty level of such information could be considered sub-optimal, its proper aggregation for multiple experts from diverse domains has the potential to reduce epistemic uncertainty. This could therefore increase the reliability of forecasts on promising technologies without prior experimental data. Such advantages could be achieved by allowing the experts to define their evaluations as fuzzy sets.

\subsection{Definition of the Main Method Parameters}

To this day, multiple implementations of Fuzzy AHP (FAHP) have been introduced in the literature, such as the approach of Laarhoven and Pedrycz, as well as the one of Buckley [28,33]. Since the latter overcomes some drawbacks of the former one [28], is more intuitive and allows the use of trapezoidal fuzzy numbers, Buckley's method is used to integrate fuzzy sets into AMA at this stage.

Before introducing its actual methodology within the AMA, a short overview of the parameters will be given; these need to be set when defining an FAHP method for a particular task. According to Liu et al. [29], these are:

1. Choice of appropriate fuzzy set types as a "representation of the relative importance for pairwise comparison" [29] (p. 2);

2. Techniques for fuzzy set aggregation when multiple experts are involved in the evaluation;

3. "Defuzzification of a fuzzy set to a crisp value for final comparison" [29] (p. 2);

4. Techniques for the consistency check of the fuzzy evaluation matrix.

As already stated in Section 2.4, ordinary fuzzy sets of Type 1 in the form of trapezoidal fuzzy numbers have been selected for the initial consideration of uncertainties. Since the current paper aims to outline the global methodology of the FAHP integration into the AMA, the rest of the parameters will be left to be set in a further, more detailed definition of the method. 


\subsection{Representation of the Aerospace Vehicle Conceptual Design Problem as a Hierarchy}

The FAHP is initialized by defining the given multi-criteria problem statement as a hierarchical tree. By following the logic outlined in Section 3.2, the attributes and the options of a given MM are used to form a comprehensible hierarchy. In this context, the current work suggests a hierarchy structure for the conceptual design of aerospace vehicles by using the attributes and options from the $\mathrm{MM}$ and the evaluation criteria. An example hierarchy for a sample aircraft design task is presented in Figure 8.

Since the technological options are the smallest system elements in the current representation, these are positioned at the lowest hierarchy level. The quality of each is evaluated with respect to all criteria, defined in level 3 above. These might be qualities such as Operating Empty Weight (OEW), the aerodynamic qualities of the aircraft (L/D or "L over D ratio"), Direct Operating Cost (DOC), different types of emissions, etc. Next, one obtains the importance of each criterion for the corresponding system attribute (level 2). Finally, the experts will give their priorities for the separate attributes regarding the global aircraft concept set as the ultimate hierarchy goal.

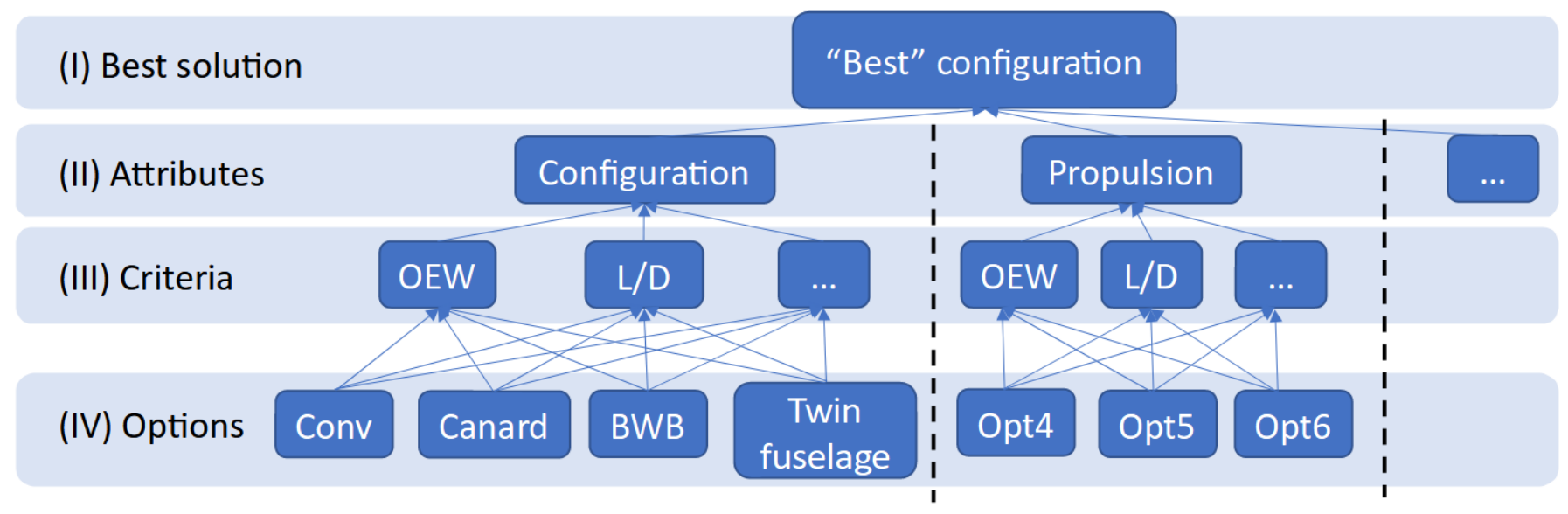

Figure 8. Suggested hierarchy structure for the evaluation process of aircraft system elements and their technological options. Abbreviations: OEW-Operating Empty Weight; L/D—“L over D" or the aerodynamic qualities; Conv—conventional configuration; BWB-Blended Wing Body; Opt—potential additional options.

One should highlight that the different attributes and their corresponding children elements are represented as separate branches in the hierarchy, which do not interconnect. This consideration was necessary due to the fact that the AHP implies pairwise comparison evaluations of elements from the same level. In this context, one cannot compare technological options for different attributes (e.g., electric propulsion and the conventional aircraft configuration) or their assigned criteria (e.g., the weight of the configuration and the weight of the propulsion subsystem).

It is important to underline that the advantage of the AHP is that the hierarchy structure is not fixed by the methodology and can be adapted according to the needs of the given problem statement. Hence, the presented hierarchy definition does not pretend to be optimal for every problematic. However, it is suggested as a structured approach to extend the MA and aid in the option evaluation, particularly in the domain of aerospace vehicle conceptual design.

\subsection{Evaluation of Hierarchy Elements with Fuzzy Sets}

The most commonly used fuzzy scales for pairwise importance comparisons are the nine- and five-level ones [29] (p. 16). In some studies, researchers fix the fuzzy numbers denoting the varying quality. In such cases, an exemplary nine-level scale would contain triangular fuzzy numbers with pre-defined peaks of full membership for the importance of 
one alternative over the other such as: equal-(1,1,2); weak-(2,3,4), strong-(4,5,6); significant$(6,7,8)$; absolute- $(8,9,9)[29]$. Here, the $(a, b, c)$ format denotes the significant points of a triangular fuzzy number, where $b$ is the peak with full membership of 1 and $a$ and $c$ are the limits of the number with membership 0 and are connected linearly to $b$.

However, the aim of the AMA enhancement is to give the experts more freedom in the representation of their opinions, rather than just providing them with a fixed set of pre-defined fuzzy numbers. Therefore, a nine-degree scale is suggested, where each degree is assigned a linguistic meaning. The experts are then allowed to express their preferences by freely defining and positioning a trapezoidal fuzzy number per evaluation. Figure 9 shows a single exemplary expert evaluation stating that technology A is "approximately from strongly to significantly superior" to technology B.

\section{Evaluation task:}

\section{How much superior is Technology $A$ in reference}

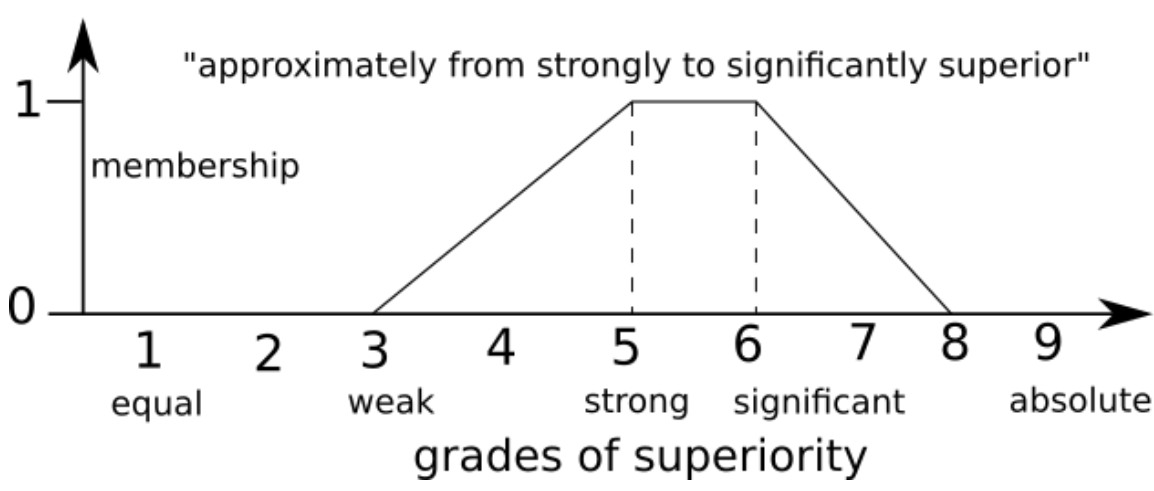

Figure 9. Suggested approach to evaluate the pairwise comparison of technological option A to option B with respect to a certain criterion.

The presented scale so far describes only the superiority degree of one option over another. As mentioned in Section 3.2, the classical crisp AHP defines option inferiority as the corresponding reciprocal crisp number. For the FAHP, Buckley $[28,33]$ represents the evaluations of inferior options by mirroring the base values of the trapezoidal fuzzy number and taking their reciprocal values [28] (p. 359). For example, if the superiority of technology A over technology B is defined by the trapezoidal fuzzy number $\tilde{a}_{A B}=(3,5,6,8)$, as shown in Figure 9, then the inferiority of technology B to technology A would be $\tilde{a}_{B A}=(1 / 8,1 / 6,1 / 5,1 / 3)$. One could also use the same nine-degree scale for the expert evaluation task. However, the expert would need to specify whether the current evaluation is about an inferior option, so that the reciprocal fuzzy number is taken afterwards.

Based on the defined hierarchy structure, the experts give their pairwise relative evaluations for each element of the corresponding hierarchy level. Consider the example hierarchy presented in Figure 8. The evaluations made by a single expert of the Blended Wing Body (BWB) configuration in comparison to the other configurations (same hierarchy level) and with respect to the aircraft OEW could be as shown in Figure 10.

These evaluations are obtained for each element of each corresponding level in order to be placed in the comparison matrices. Equation (5) shows the structure of a reciprocal comparison matrix, where $a_{i j k}$ is the fuzzy trapezoidal comparison of option $i$ with option $j$ made by the $k$-th expert (for the total of $n$ options and $p$ elements) [28].

$$
D=\left[\begin{array}{cccc}
(1,1,1,1) & a_{121} ; a_{122} ; \ldots ; a_{12 p} & \ldots & a_{1 n 1} ; a_{1 n 2} ; \ldots ; a_{1 n p} \\
\ldots & \ldots & \ldots & \ldots \\
\ldots & a_{i j 1} ; a_{i j 2} ; \ldots ; a_{i j p} & \ldots & \ldots \\
\ldots & \ldots & \ldots & \ldots \\
a_{n 11} ; a_{n 12} ; \ldots ; a_{n 1 p} & a_{n 21} ; a_{n 22} ; \ldots ; a_{n 2 p} & \ldots & (1,1,1,1)
\end{array}\right]
$$


Subsequently, the evaluations of all experts should be aggregated for each option. As previously mentioned, the choice of the most appropriate aggregation method is left as a topic for future investigations.

\section{Evaluation task:}

\section{How much superior is the Blended-Wing-Body
configuration in respect to OEW?}

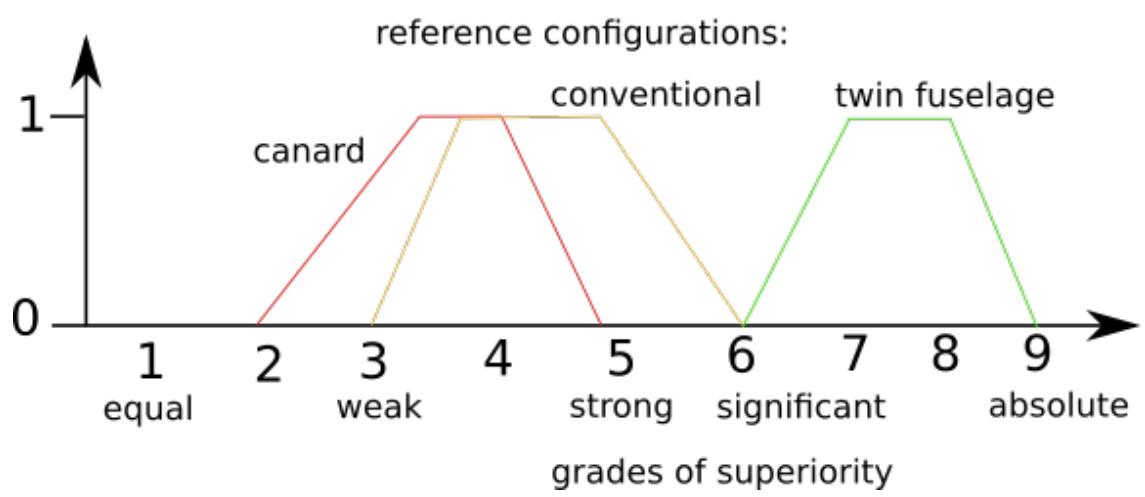

Figure 10. Example of pairwise comparisons made by a single expert of the BWB configuration referred to the configurations twin fuselage, canard and the conventional one, with respect to the OEW criterion.

\subsection{Buckley's Approach for the Implementation of Fuzzy Analytic Hierarchy Process}

Once all aggregated evaluations have been acquired and organized into the corresponding comparison matrices, Buckley's FAHP approach can be applied to obtain the global weights of the options [28] (p. 356). This is outlined in the following steps [28]:

(1) Calculation of the fuzzy weights $\omega_{i}$ for the elements of the same hierarchy level.

For this purpose, the geometric mean $z_{i}$ for each row (corresponding to option $i$ ) of the reciprocal comparison matrix is estimated first (Equation (6)). The fuzzy weight $\omega_{i}$ is then calculated by referring the corresponding geometric mean of the row $z_{i}$ to the sum of the geometric means in all rows, as shown in Equation (7). The signs $\oplus$ and $\odot$ stand for fuzzy addition and fuzzy multiplication, as defined in [28] (pp. 353-356), respectively.

$$
\begin{gathered}
z_{i}=\left(a_{i 1} \odot \ldots \odot a_{i n}\right)^{1 / n} \\
\omega_{i}=z_{i} \odot\left(z_{1} \oplus \ldots \oplus z_{n}\right)^{-1}
\end{gathered}
$$

(2) Estimation of the global importance of the options over all hierarchy levels. This is achieved by following the logic of the original crisp AHP. The importance of each $i$-th option $U_{i}$ is derived as the sum of the option weights $\omega_{i, j}$ each multiplied by the weight $b_{j}$ of the corresponding element $j$ from the higher level of the hierarchy.

$$
U_{i}=\sum_{j=1}^{n} b_{j} \omega_{i j}
$$

\section{Discussion}

Along with the detailed descriptions of the presented methods in the sections above, it is necessary to underline the novelties suggested in the current article for the field of conceptual aircraft design. Furthermore, the findings will be positioned within the context of the existing literature on the subject.

The choice of the most suitable configuration within the AMA depends on the evaluations of technological options by a group of dedicated experts. Thus far, this process has been defined solely through the structure of the morphological matrix, namely the system attributes and their corresponding technological options, as well as by the evaluation 
criteria. The evaluations' reliability can be increased not only by introducing uncertainty modeling, but also by representing the complex problem definitions and the evaluation process in a structured way. For this purpose, the Fuzzy Analytic Hierarchy Process has been adapted for its use in the AMA. This was possible through the suggested novel definition of the aerospace vehicle design problem as an intuitive hierarchy structure, a concrete format for potential evaluation questions as well as an appropriate fuzzy evaluation scale.

The improved organization of criteria intuitively follows the levels of importance of attributes and subsystems. This logical yet flexible structure not only allows us to conduct the evaluation process in a formalized order; it also gives the experts the opportunity to discuss whole groups of alternatives from the same hierarchy level simultaneously. This makes it possible to widen the discussion and take into account aspects that could be left out when evaluating each option separately.

It is also important to position the findings among existing sources on the use of fuzzy sets and MADM or (F)AHP methods in the aviation domain. Thus far, these have been applied to abstract and non-deterministic problems mostly in the field of aeronautical operations or to answer organizational questions. The methods have been used, e.g., for the assessment of technical factors in aviation safety [34], the evaluation and ranking of green airlines [35], the assessment of factors to reduce fuel consumption [36] and the evaluation of aircraft efficiency [37]. Additionally, AHP has been applied to develop advanced brainstorming methods for the design of novel aircraft configurations, e.g., cryogenic planes in [38].

However, there is still a gap in the systematic idea generation and analysis of alternative aerospace vehicle concepts, particularly when using innovative technologies with dominating uncertainties. Therefore, the current article addresses these challenges by extending the AMA.

At this point, it is necessary to outline recommendations in order to continue the purposeful AMA enhancement in the specified direction. Firstly, the presented uncertainty modeling through fuzzy trapezoidal numbers and the given hierarchy structures should be integrated into a full-scale structured expert judgment elicitation framework. This will define in full detail the planning, conduction and evaluation of the expert workshops by using the presented novel approach. Secondly, a study should be conducted for the purpose of choosing the proper aggregation method for fuzzy evaluations by different experts. In this context, one could also consider the professional background of each expert, which could justify a certain weighting scheme for the marks given by experts from different domains. Furthermore, a strategy should be found to consider possible interactions of technological options contributing to the overall solution evaluation. Finally, in the event that the necessity for the deeper modeling of uncertainties arises, one could study the use of other fuzzy number types instead of the ordinary Type 1 ones.

\section{Conclusions}

There are currently multiple limitations and challenges during the conceptual design phase of innovative aerospace vehicles, particularly in the context of idea generation and concept evaluation. In this context, the Advanced Morphological Approach still exhibits the methodological gaps of dominating uncertainties and the lack of robust structuring for complex engineering solutions. The current article suggests concrete approaches to model uncertainties via fuzzy sets, structure the conceptual design problematic and formalize the evaluation of technological alternatives. The proposed methods have the potential to offer a new level of reliability for the decisions on innovative technologies made during the conceptual design phase in aerospace.

Existing taxonomies of uncertainties have been studied in order to identify the uncertainty types present in the AMA process. During the evaluations of technological options, one faces unmeasurable epistemic uncertainties, which are mostly due to the lack of statistical data and expert knowledge on untested innovative technologies. After outlining multiple approaches for uncertainty modeling, this work justifies the use of fuzzy sets for 
the representation of epistemic uncertainty within aerospace conceptual design. In particular, a suggestion has been put forward to represent unsharp evaluation statements made by experts through Type 1 trapezoidal fuzzy numbers.

Furthermore, the Fuzzy Analytical Hierarchy process has been chosen and integrated into the AMA as a method to structure the evaluation of technological alternatives. In this context, the conceptual design problematic of aerospace vehicles has been defined as a hierarchical structure, which intuitively reflects the levels of evaluation criteria. In order to justify the practical applicability of the presented approach, the current work suggests a concrete format for the evaluation questions, as well as an appropriate fuzzy scale to capture the experts' uncertainty.

The presented enhancements of the AMA method have the potential to improve the reliability of aerospace concept evaluations, especially when innovative and untested technologies are involved. Therefore, it is a step towards giving the designer more independence from already proven concepts and increasing confidence in the consideration of unconventional aerospace configurations.

The suggested methodology is defined as one of the integral stages necessary for the development of a much wider design method. The complex and abstract nature of the whole approach requires the thorough definition of further AMA improvements, which were outlined in the Section 5.

Not only does the proposed approach represent one of the multiple AMA improvement stages, but it could also be used as a "stand-alone" methodological component for similar research purposes in the aerospace domain and beyond. The authors' vision is the practical application of the improved AMA in the aerospace industry as well as in other fields with demand for non-deterministic conceptual product design. In order to ensure its smooth and intuitive application in the design process, it is planned to implement the complex logic and operations of the novel method into a software tool with formalized workshop instructions. In addition to the advantages of the AMA itself, the workshops using such a design framework would allow the experts to intuitively evaluate the technology alternatives by automatically accounting for uncertainties and reducing cognitive biases. Finally, the ultimate goal is to serve as a reliable decision-making aid when choosing the optimal concept.

Author Contributions: Conceptualization, V.T.T., D.R. and A.B.; Funding acquisition, A.B.; Investigation, V.T.T. and D.R.; Methodology, V.T.T. and D.R.; Project administration, A.B.; Resources, D.R. and A.B.; Supervision, D.R. and A.B.; Visualization, V.T.T. and D.R.; Writing-original draft, V.T.T.; Writing - review and editing, D.R. and A.B. All authors have read and agreed to the published version of the manuscript.

Funding: This research was funded by Deutsche Forschungsgemeinschaft (DFG, German Research Foundation), project number 443831887.

Conflicts of Interest: The authors declare no conflict of interest.

\section{Abbreviations}

The following abbreviations are used in this manuscript:

$\begin{array}{ll}\text { MA } & \text { Morphological Analysis } \\ \text { AMA } & \text { Advanced Morphological Analysis } \\ \text { MM } & \text { Morphological Matrix } \\ \text { Attr } & \text { Attribute } \\ \text { Opt } & \text { Option } \\ \text { DM } & \text { Decision-Maker } \\ \text { PDF } & \text { Probability Density Function } \\ \text { MCDM } & \text { Multi-Criteria Decision-Making } \\ \text { MODM } & \text { Multiple-Objective Decision-Making } \\ \text { MADM } & \text { Multiple-Attribute Decision-Making }\end{array}$




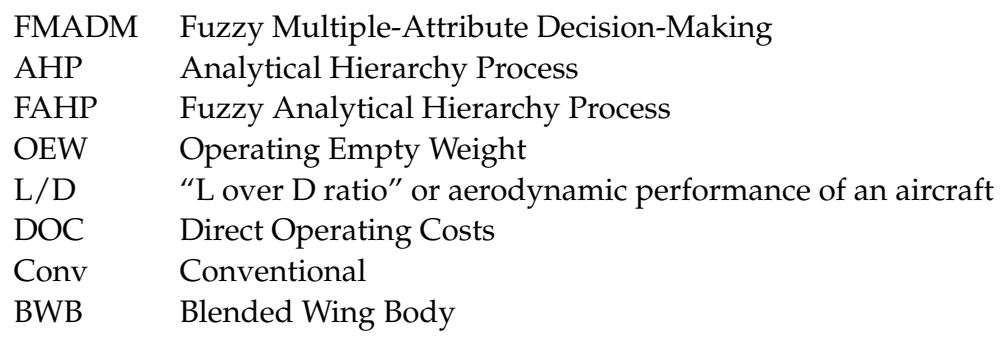

\section{References}

1. Fielding, J.P. Introduction to Aircraft Design, 2nd ed.; Cambridge University Press: Cambridge, UK, 2017. [CrossRef]

2. Rand, O.; Khromov, V. Mission Oriented Multi-Prop UAV Analysis Using Statistical Design Trends. Aerospace 2021, 8, 321. [CrossRef]

3. Mishin, V.P.; Osin, M.I. Introduction to Aircrafts Machine Design; Mashinostroenie: Moscow, Russia, 1978.

4. European Commission; Directorate General for Research and Innovation; Directorate General for Mobility and Transport. Flightpath 2050: Europe's Vision for Aviation: Maintaining Global Leadership and Serving Society's Needs; Publications Office of the European Union: Luxembourg, 2011.

5. The Shift Project; SUPAERO DECARBO. Pouvoir voler en 2050: Quelle Aviation dans un Monde Contraint? (Flying in 2050: What Future for Aviation in a Constrained World?); Technical Report. Available online: https://theshiftproject.org/article/ quelle-aviation-dans-un-monde-contraint-nouveau-rapport-du-shift/ (access on 3 March 2021).

6. Sadraey, M.H. Aircraft Design: A Systems Engineering Approach; Aerospace Series; Wiley: Chichestet, UK, 2013.

7. Bardenhagen, A.; Rakov, D. Analysis and Synthesis of Aircraft Configurations during Conceptual Design Using an Advanced Morphological Approach. Deutsche Gesellschaft für Luft- und Raumfahrt-Lilienthal-Oberth e.V. Available online: https: / / publikationen.dglr.de/?tx_dglrpublications_pi1\%5bdocument_id\%5d=490063 (accessed on 6 November 2019). [CrossRef]

8. Bardenhagen, A.; Rakov, D. Advanced Morphological Approach in Aerospace Design During Conceptual Stage. Facta Univ. Ser Mech. Eng. 2019, 17, 321-332. [CrossRef]

9. Klimenko, B.; Rakov, D. Analysis and Synthesis of Innovative Engineering Solutions and Technologies Based on Advanced Morphological Approach. In Advances in Artificial Systems for Medicine and Education; Advances in Intelligent Systems and Computing; Hu, Z., Petoukhov, S., He, M., Eds.; Springer International Publishing: Cham, Switzerland, 2018; Volume 658, pp. 274-283. [CrossRef]

10. Rakov, D. Morphological synthesis method of search for promising technical system. IEEE Aerosp. Electron. Syst. Mag. 1996, 11, 3-8. [CrossRef]

11. Rakov, D. Superlight Reentry Vehicles. Space Technol. 2004, 24, 237-243.

12. Todorov, V.T.; Rakov, D.; Bardenhagen, A. Creation of Innovative Concepts in Aerospace Based on the Morphological Approach. In Proceedings of the 11th EASN Virtual International Conference on Innovation in Aviation \& Space to the Satisfaction of the European Citizens, Salerno, Italy, 1-3 September 2021.

13. Roelofs, M.; Vos, R. Uncertainty-Based Design Optimization and Technology Evaluation: A Review. In Proceedings of the 2018 AIAA Aerospace Sciences Meeting, Kissimmee, FL, USA, 8-12 January 2018; American Institute of Aeronautics and Astronautics: Kissimmee, FL, USA, 2018. [CrossRef]

14. Yao, W.; Chen, X.; Luo, W.; van Tooren, M.; Guo, J. Review of uncertainty-based multidisciplinary design optimization methods for aerospace vehicles. Prog. Aerosp. Sci. 2011, 47, 450-479. [CrossRef]

15. Thunnissen, D.P. Propagating and Mitigating Uncertainty in the Design of Complex Multidisciplinary Systems. Ph.D. Thesis, California Institute of Technology: Pasadena, CA, USA, 2005. [CrossRef]

16. Tversky, A.; Kahneman, D. Judgment under Uncertainty: Heuristics and Biases. Science 1974, 185, 1124-1131. [CrossRef] [PubMed]

17. Zang, T.A.; Hemsch, M.J.; Hilburger, M.W.; Kenny, S.P.; Luckring, J.M.; Maghami, P.; Padula, S.L.; Stroud, W.J. Needs and Opportunities for Uncertainty-Based Multidisciplinary Design Methods for Aerospace Vehicles; Technical Memorandum (TM) NASA/TM2002-211462; Issue: NASA/TM-2002-211462; Langley Research Center, National Aeronautics and Space Administration: Hampton, VA, USA, 2002.

18. Oberkampf, W.; Helton, J.; Sentz, K. Mathematical representation of uncertainty. In Proceedings of the 19th AIAA Applied Aerodynamics Conference, Anaheim, CA, USA, 11-14 June 2001; American Institute of Aeronautics and Astronautics: Anaheim, CA, USA, 2001. [CrossRef]

19. Monroe, R.W. A Synthesized Methodology for Eliciting Expert Judgment for Addressing Uncertainty in Decision Analysis. Ph.D. Thesis, Old Dominion University Libraries, Norfolk, VA, USA, 1997. [CrossRef]

20. Unal, R.; Keating, C.; Conway, B.; Chytka, T. Development of an Expert Judgement Elicitation Methodology Using Calibration and Aggregation for Risk Analysis in Conceptual Vehicle Design; Technical Report 20040016143; Issue: 20040016143; NASA Langley Research Center, Engineering Management Department Old Dominion University: Norfolk, VA, USA, 2004.

21. Zadeh, L. The concept of a linguistic variable and its application to approximate reasoning-I. Inf. Sci. 1975, 8, 199-249. [CrossRef]

22. Dubois, D.; Prade, H. Fundamentals of Fuzzy Sets; Springer: Boston, MA, USA, 2000. 
23. Kahraman, C.; Öztaysi, B.; Onar, S.C. A Comprehensive Literature Review of 50 Years of Fuzzy Set Theory. Int. J. Comput. Intell. Syst. 2016, 9, 3. [CrossRef]

24. Atanassov, K.T. Intuitionistic fuzzy sets. Fuzzy Sets Syst. 1986, 20, 87-96. [CrossRef]

25. Szmidt, E.; Kacprzyk, J. A consensus-reaching process under intuitionistic fuzzy preference relations. Int. J. Intell. Syst. 2003, 18, 837-852. [CrossRef]

26. Torra, V. Hesitant fuzzy sets. Int. J. Intell. Syst. 2010, 25, 529-539. [CrossRef]

27. Kahraman, C.; Onar, S.C.; Oztaysi, B. Fuzzy Multicriteria Decision-Making: A Literature Review. Int. J. Comput. Intell. Syst. 2015, 8, 637-666. [CrossRef]

28. Chen, S.J.; Hwang, C.L.; Hwang, F.P. Fuzzy Multiple Attribute Decision Making: Methods And Applications; Number 375 in Lecture Notes in Economics and Mathematical Systems; Springer: Berlin, Germany; New York, NY, USA, 1992.

29. Liu, Y.; Eckert, C.M.; Earl, C. A review of fuzzy AHP methods for decision-making with subjective judgements. Expert Syst. Appl. 2020, 161, 113738. [CrossRef]

30. Mota, P.; Campos, A.R.; Neves-Silva, R. First Look at MCDM: Choosing a Decision Method. Adv. Smart Syst. Res. 2013, 3, 25-30.

31. Figueira, J.; Greco, S.; Ehrgott, M. (Eds.) Multiple Criteria Decision Analysis: State of the Art Surveys; Springer: New York, NY, USA, 2005.

32. Saaty, T. Decision-Making. Analytic Hierarchy Process; Radio i Svyaz: Moscow, Russia, 1993.

33. Buckley, J. Fuzzy hierarchical analysis. Fuzzy Sets Syst. 1985, 17, 233-247. [CrossRef]

34. Chen, C.J.; Yang, S.M.; Chang, S.C. A model integrating fuzzy AHP with QFD for assessing technical factors in aviation safety. Int. J. Mach. Learn. Cybern. 2014, 5, 761-774. [CrossRef]

35. Alkhatib, S.F.; Migdadi, Y.K.A.A. A novel technique for evaluating and ranking green airlines: Benchmarking-base comparison. Manag. Environ. Qual. Int. J. 2020, 32, 210-226. [CrossRef]

36. Singh, J.; Sharma, S.K.; Srivastava, R. AHP-Entropy based priority assessment of factors to reduce aviation fuel consumption. Int. J. Syst. Assur. Eng. Manag. 2019, 10, 212-227. [CrossRef]

37. Grzesik, N. Fuzzy sets in aircraft system efficiency evaluation. Aircr. Eng. Aerosp. Technol. 2016, 88, 707-716. [CrossRef]

38. Ucler, C. Brainstorming the cryoplane layout by using the iterative AHP-QFD-AHP approach. Aviation 2018, 21, 55-63. [CrossRef] 